\title{
Feasibility and Acceptability of Community Coronavirus Disease 2019 Testing Strategies (FACTS) in a University Setting
}

\author{
Jennifer A. Hirst, ${ }^{1,2, a, \odot}$ Mary Logan, ${ }^{1,2, a, \odot}$ Thomas R. Fanshawe, ${ }^{1}$ Lazaro Mwandigha, ${ }^{1}$ Marta Wanat, ${ }^{1}$ Charles Vicary, ${ }^{1}$ Rafael Perera, ${ }^{1,2}$ \\ Sarah Tonkin-Crine, ${ }^{1,3, \odot}$ Joseph Jonathan Lee, ${ }^{1, \odot}$ Irene Tracey, ${ }^{4,5}$ Gordon Duff, ${ }^{6}$ Peter Tufano, ${ }^{7}$ Marya Besharov, ${ }^{7}$ Lionel Tarassenko, ${ }^{8,9}$ Brian D. Nicholson, \\ and F. D. Richard Hobbs ${ }^{1,2}$ \\ ${ }^{1}$ Nuffield Department of Primary Care Health Sciences, University of Oxford, Radcliffe Observatory Quarter, Oxford, United Kingdom, ${ }^{2}$ National Institute for Health Research Oxford Biomedical \\ Research Centre, University of Oxford Hospitals NHS Foundation Trust, Oxford, United Kingdom, ${ }^{3}$ National Institutes of Health Research Health Protection Research Unit in Healthcare Associated \\ Infections and Antimicrobial Resistance, University of Oxford, Oxford, United Kingdom, ${ }^{4}$ Nuffield Department of Clinical Neurosciences, John Radcliffe Hospital, Oxford, United Kingdom, ${ }^{5}$ Merton \\ College, University of Oxford, Oxford, United Kingdom, ${ }^{6}$ St Hilda's College, University of Oxford, Oxford, United Kingdom, ${ }^{7}$ Said Business School, University of Oxford, Oxford, United Kingdom, \\ ${ }^{8}$ Sensyne Health, Oxford, United Kingdom, and ${ }^{9}$ Institute of Biomedical Engineering, Department of Engineering Science, Headington, Oxford, United Kingdom
}

Background. During the coronavirus disease 2019 (COVID-19) pandemic in 2020, the UK government began a mass severe acute respiratory syndrome coronavirus 2 (SARS-CoV-2) testing program. This study aimed to determine the feasibility and acceptability of organized regular self-testing for SARS-CoV-2.

Methods. This was a mixed-methods observational cohort study in asymptomatic students and staff at University of Oxford, who performed SARS-CoV-2 antigen lateral flow self-testing. Data on uptake and adherence, acceptability, and test interpretation were collected via a smartphone app, an online survey, and qualitative interviews.

Results. Across 3 main sites, 551 participants (25\% of those invited) performed 2728 tests during a follow-up of 5.6 weeks; 447 participants $(81 \%)$ completed at least 2 tests, and $340(62 \%)$ completed at least 4 . The survey, completed by 214 participants (39\%), found that $98 \%$ of people were confident to self-test and believed self-testing to be beneficial. Acceptability of self-testing was high, with $91 \%$ of ratings being acceptable or very acceptable. A total of 2711 (99.4\%) test results were negative, 9 were positive, and 8 were inconclusive. Results from 18 qualitative interviews with students and staff revealed that participants valued regular testing, but there were concerns about test accuracy that impacted uptake and adherence.

Conclusions. This is the first study to assess feasibility and acceptability of regular SARS-CoV-2 self-testing. It provides evidence to inform recruitment for, adherence to, and acceptability of regular SARS-CoV-2 self-testing programs for asymptomatic individuals using lateral flow tests. We found that self-testing is acceptable and people were able to interpret results accurately.

Keywords. asymptomatic; COVID-19; lateral flow test; self-testing; university.

\section{KEY POINTS}

To determine feasibility and acceptability of rapid SARS-CoV-2 self-testing in a university setting, 551 participants completed 2728 lateral flow COVID-19 swab tests during a mean follow-up

Received 29 June 2021; editorial decision 22 September 2021; accepted 24 September 2021; published online 4 October 2021

J. A. H. and M. L. contributed equally to this work as joint first authors.

Correspondence: F. D. Richard Hobbs, CBE, FMedSci, FRCGP, FRCP (London), FESC, FRCP (Edin), MA (Ox), Nuffield Department of Primary Care Health Sciences, University of Oxford, Radcliffe Observatory Quarter, Woodstock Road, Oxford OX2 6GG, UK (richard.hobbs@phc. ox.ac.uk)

Open Forum Infectious Diseases ${ }^{\circledR} 2021$

(C) The Author(s) 2021. Published by Oxford University Press on behalf of Infectious Diseases Society of America. This is an Open Access article distributed under the terms of the Creative Commons Attribution-NonCommercial-NoDerivs licence (https://creativecommons.org/ licenses/by-nc-nd/4.0/), which permits non-commercial reproduction and distribution of the work, in any medium, provided the original work is not altered or transformed in any way, and that the work is properly cited. For commercial re-use, please contact journals.permissions@oup.com https://doi.org/10.1093/ofid/ofab495 of 5.6 weeks. Ninety-three percent rated testing as acceptable or very acceptable.

During coronavirus disease 2019 (COVID-19) pandemic testing for severe acute respiratory syndrome coronavirus 2 (SARS-CoV-2) in the United Kingdom (UK), laboratory-based reverse-transcription polymerase chain reaction (RT-PCR) tests focused only on those with specific symptoms. However, some infected individuals remain asymptomatic, and in symptomatic cases, viral shedding is likely to occur before symptoms develop [1]. This led to calls for population-level asymptomatic SARS-CoV-2 screening [2,3], which required a reliable, affordable testing strategy that ideally could be self-administered.

In late 2020, lateral flow tests (LFTs) were introduced for rapid detection of the SARS-CoV-2 antigen [4]. LFTs do not require laboratories, can be performed locally, and produce results within 30 minutes. In October 2020, the UK government launched a mass testing initiative using LFTs nationwide 
[5] However, one-off LFT diagnostic performance [6] does not meet the World Health Organization accepted minimum of $97 \%$ specificity and $80 \%$ sensitivity, and data on diagnostic performance from asymptomatic self-testing are sparse [7]. To improve diagnostic performance of the overall testing strategy, regular retesting was proposed [8]. Potential use of LFTs in population screening became an international research priority. Scientific advice [9] indicated that a $\geq 90 \%$ uptake and selfisolation was necessary for a successful screening strategy [10].

Student populations (typically a high proportion of young adults) are more likely to experience asymptomatic SARS-CoV-2 infection than older people [11] and the potential for transmission in university populations is significant, particularly at term start when students return from disparate locations. Modeling data suggest that very frequent repeated student asymptomatic swabbing and self-testing (every $2-3$ days) would be required to prevent SARS-CoV-2 outbreaks $[8,12]$. It was unclear, however, if this strategy would be feasible and acceptable to university students and staff as there are no published studies assessing this strategy using LFTs on a university campus.

This study's aims were to determine the feasibility and acceptability of implementing organized, regular, mass self-testing for COVID-19 in asymptomatic University of Oxford students and staff using a smartphone app and qualitative interviews. This work provides important data on wider community COVID-19 testing potential.

\section{METHODS}

\section{Design, Setting, and Participants}

Feasibility and Acceptability of Community Coronavirus Disease 2019 Testing Strategies (FACTS) is a mixed-methods cohort study conducted at the University of Oxford, approved by the University of Oxford Research Ethics Committee (CUREC ethics reference R72896/RE001 October 2020).

Eligible participants ( $\geq 16$ years of age) were those working or studying across 3 university sites. Eligibility criteria included showing no COVID-19 symptoms (new continuous cough, high temperature, and/or loss of/change to sense of smell/taste) and being capable of LFT self-administration following training.

\section{Recruitment and Follow-up}

Two colleges began recruitment on 29 October 2020 and 1 department began recruitment on 17 November 2020 (sites A, B, and C). In late November, recruitment was extended across the university, but stopped in early December when a universitywide COVID-19 screening program was implemented, leaving only sites A, B, and C continuing with FACTS. Invitations to participate and study information were sent by email, first only to students and later to staff.
To participate, individuals download a free app (for iOS or Android operating systems, "CVm-Health+ Education," developed by Sensyne Health PLC, Oxford, UK), to their smartphones; had the option to consent, indicated willingness to be contacted for interview, and provided an email address, sex/ gender, date of birth, and ethnicity.

Trainers supervised participants' first test during face-to-face or online training. Electronic training materials and instructions were also available. Participants got an LFT kit, which they used to self-swab and test. After 30 minutes, they interpreted results as negative, positive, or inconclusive (failed test) and uploaded a photograph of the result to the app. Inconclusive tests were repeated using the remaining sample and a new test cartridge. Acceptability of conducting the test was rated on a 1-5 scale ("very acceptable" to "very unacceptable"). The full procedure is shown in Supplementary Appendix 1.

If COVID-19-related symptoms developed or if an LFT result was positive, participants were instructed to self-isolate and book an RT-PCR test. Participants were also asked to upload their health status daily to track development of any COVID19-related symptoms.

Repeat weekly testing was performed at a central location on sites A, B, and C until late November. Thereafter, participants were supplied with kits to continue testing at home throughout Christmas until 18 January 2021 (study end date). Follow-up did not take place at other recruiting sites. Because of these differences in follow-up potential, all sites other than A, B, and C are grouped together as "other sites."

If a participant received a COVID-19-positive RT-PCR test result, they were asked to stop self-testing. Participants could withdraw from the study without providing a reason.

\section{Lateral Flow Test}

The Innova Rapid SARS-CoV-2 Antigen Test Kit (Innova Medical Group), developed for testing symptomatic individuals, was the LFT used. A positive test result is given within 20 minutes, a negative within 30 minutes [13].

\section{Data Collection and Analysis}

The CVm-Health+ Education app collected all quantitative data. Analyses were restricted to consented participants who uploaded $\geq 1$ LFT result photograph. Recruitment, follow-up (time between first and last submitted test), and demographics were summarized as numbers and percentages, or mean and standard deviation (SD). Patterns of test results for participants who reported a positive LFT or RT-PCR result were shown graphically. LFT reporting accuracy was assessed by comparing the participant-reported result with the uploaded LFT photograph. App-reported symptom prevalence was summarized and results were broken down for the 3 main sites. Analyses were conducted using the statistical software package R. 


\section{Mixed-Methods Evaluation}

A mixed-methods evaluation involving surveys and interviews was nested within the study.

The survey assessed participants' views on testing benefits; barriers to regular testing; trust in test results; and intentions to continue testing, act on a positive result, and self-isolate if indicated (Supplementary Appendix 2). Survey items were designed for responses on a 7-point Likert scale, ranging from "strongly disagree" to "strongly agree." Additional information collected included type of training received, university role, and whether symptoms were experienced during study participation. A free-text box was included for comments on any aspect of the testing experience. The survey was designed using the Jisc Online survey platform. All participants received an email with a survey web link on 1 December 2020. Quantitative survey data were analyzed using descriptive statistics.

Participants $(\mathrm{n}=18$ ) were invited for interview using purposive sampling. Following consent, telephone/online interviews were conducted and audio was recorded. Interviews continued until data indicated saturation [14]. Interviews explored views and experiences of test use, regular testing barriers and facilitators, trust in test results, perceived testing benefits, and intentions to act on positive result (Supplementary Appendix 3, Topic Guide). We conducted rapid data collection and analysis concurrently [15]. Using the survey's free-text comments, we created a framework used for interview analysis. This method was deemed a pragmatic and efficient approach to collect and analyze data rapidly during a public health emergency [15].

\section{RESULTS}

\section{Recruitment and Testing}

At the 3 primary recruitment sites (A, B, and C), 2195 students and staff were eligible, 664 (31\%) attended training, and 551 (25\%) participated (Figure 1). An additional 183 participants were recruited across other sites, so the total sample size was 734. One hundred thirteen participants who attended training were ineligible as no LFT result photograph was uploaded. Eighty percent $(n=588)$ of participants were students, with a mean age of $26(\mathrm{SD}, 8)$ years; $20 \%(\mathrm{n}=146)$ were staff, with a mean age of 42 (SD, 11) years. Overall, 55\% were women and $83 \%$ were white. All demographics are presented in Table 1, stratified by recruitment site.

In the 3 primary sites, participants completed 2728 tests, with 447 (81\%) completing $\geq 2$ tests and 340 (62\%) completing $\geq 4$ tests. One hundred fifty-five (28\%) stopped testing after just 2 tests. The number of tests completed was slightly higher in staff than students ( 6.0 vs 4.7 , respectively). At the 3 primary sites, mean follow-up time was $5.6(\mathrm{SD}, 3.2)$ weeks and participants completed a mean of 5.0 tests each. The rate of testing (number of tests from recruitment to study end) was higher in staff ( 0.81 in staff vs 0.60 in students) (Table 1). Across all sites, 3187 LFT results were reported, with a mean of $4.3(\mathrm{SD}, 2.9)$ tests per participant. Figure 2 shows the timing of recruitment and testing over the study period.

\section{Test Results}

Of LFT results reported, 3170 (99.5\%) were negative (2711 in the 3 primary sites), 9 (in 8 participants) were positive, and 8

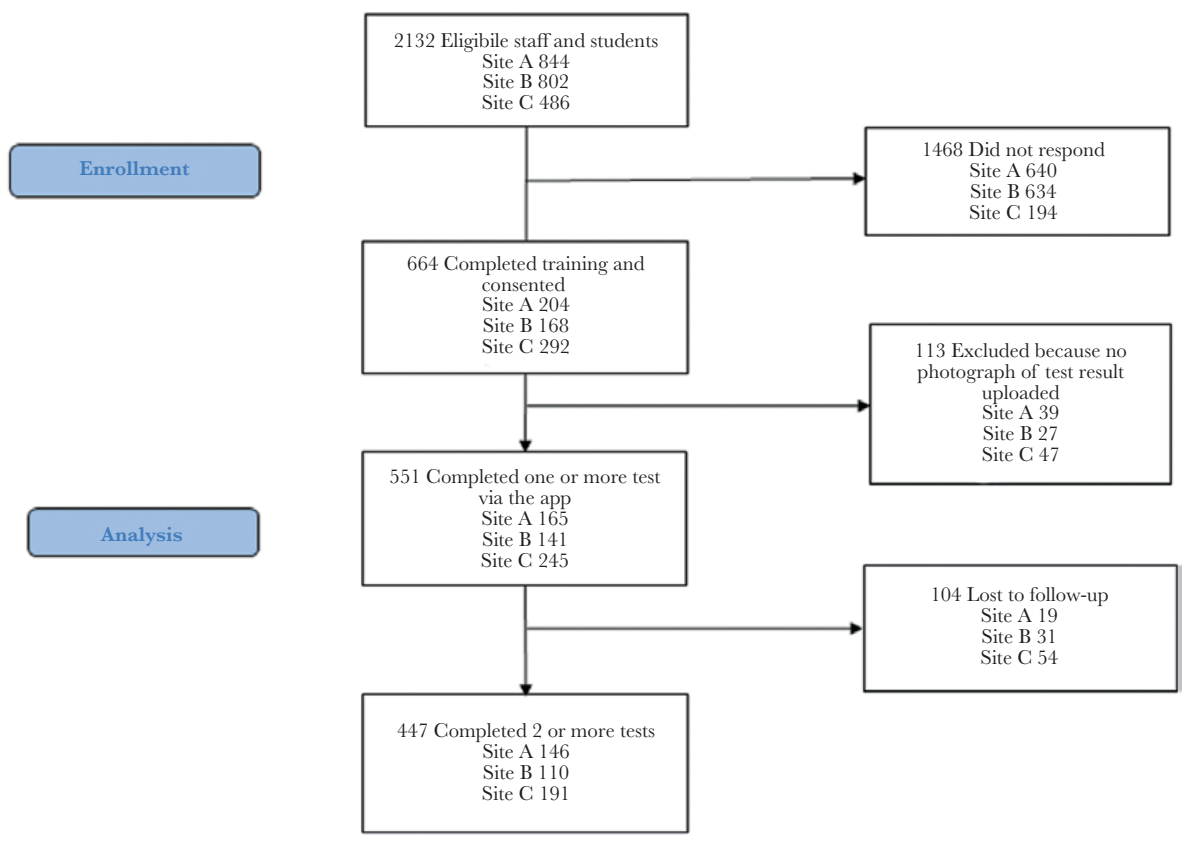

Figure 1. Participant flowchart for the 3 primary recruitment sites. 


\begin{tabular}{|c|c|c|c|c|c|c|}
\hline \multirow[b]{2}{*}{ Characteristic } & \multirow[b]{2}{*}{ Overall } & \multicolumn{4}{|c|}{ Three Main Sites } & \multirow[b]{2}{*}{ Other Sites } \\
\hline & & Combined Main Sites & Site A & Site B & Site C & \\
\hline Total No. of students and staff & $\ldots$ & 2132 & 844 & 802 & 486 & $\ldots$ \\
\hline Enrolled & $\ldots$ & $664(31 \%)$ & $204(24 \%)$ & $168(21 \%)$ & $292(60 \%)$ & $\ldots$ \\
\hline $\begin{array}{l}\text { Participants with at least } 1 \text { test } \\
\text { upload }\end{array}$ & 734 & $551(26 \%)$ & $165(20 \%)$ & $141(18 \%)$ & $245(50 \%)$ & 183 \\
\hline Staff & 146 & 115 & 31 & 23 & 61 & 31 \\
\hline Students & 588 & 436 & 134 & 118 & 184 & 152 \\
\hline No. of tests & 3187 & 2728 & 1047 & 690 & 991 & 459 \\
\hline Male sex & $327(45 \%)$ & $244(44 \%)$ & $64(39 \%)$ & $56(40 \%)$ & $124(51 \%)$ & $83(45 \%)$ \\
\hline Age, $y$, mean (SD) & $28.8(10.7)$ & $29.3(10.7)$ & $27.4(12.0)$ & $25.4(10.4)$ & $32.7(8.6)$ & $27.5(10.7)$ \\
\hline Staff & $42.2(11.0)$ & $42.1(10.8)$ & $41.8(11.7)$ & $43.3(10.7)$ & $41.8(10.5)$ & $42.7(11.6)$ \\
\hline Students & $25.5(7.7)$ & $25.9(7.6)$ & $24.1(9.3)$ & $21.9(5.7)$ & $29.7(5.1)$ & $24.4(7.3)$ \\
\hline White race & $608(83 \%)$ & $451(82 \%)$ & $150(91 \%)$ & $131(93 \%)$ & $170(69 \%)$ & $157(86 \%)$ \\
\hline $\begin{array}{l}\text { No. of tests per participant, mean } \\
\text { (SD) [range] }\end{array}$ & $4.3(2.9)[1-13]$ & $5.0(3.0)[1-13]$ & $6.3(3.0)[1-13]$ & $4.9(3.2)[1-11]$ & $4.1(2.5)[1-10]$ & $2.5(1.6)[1-9]$ \\
\hline Staff & $5.4(2.9)[1-10]$ & $6.0(2.7)[1-10]$ & $7.1(2.5)[1-10]$ & $6.9(3.0)[1-10]$ & $5.1(2.4)[1-9]$ & $3.4(2.4)[1-9]$ \\
\hline Students & $4.1(2.9)[1-13]$ & $4.7(3.0)[1-13]$ & $6.2(3.0)[1-13]$ & $4.5(3.1)[1-10]$ & $3.7(2.4)[1-10]$ & $2.3(1.4)[1-8]$ \\
\hline \multicolumn{7}{|l|}{ No. with: } \\
\hline 1 test & 137 & 104 & 19 & 31 & 54 & 33 \\
\hline 2 tests & 159 & 51 & 5 & 18 & 28 & 108 \\
\hline 3 tests & 67 & 56 & 11 & 11 & 34 & 11 \\
\hline$\geq 4$ tests & 371 & 340 & 130 & 81 & 129 & 31 \\
\hline $\begin{array}{l}\text { Rate of testing (No. of tests/weeks } \\
\text { in study) }\end{array}$ & 0.96 & 0.91 & 0.91 & 0.90 & 0.93 & 1.10 \\
\hline Staff & 0.94 & 0.93 & 0.92 & 0.97 & 0.92 & 0.99 \\
\hline Students & 0.96 & 0.91 & 0.90 & 0.88 & 0.93 & 1.12 \\
\hline $\begin{array}{l}\text { Rate of testing (No. of tests/weeks } \\
\text { from enrollment to } 18 \text { Jan 2021) }\end{array}$ & 0.56 & 0.64 & 0.72 & 0.58 & 0.63 & 0.33 \\
\hline Staff & 0.73 & 0.81 & 0.85 & 0.82 & 0.79 & 0.42 \\
\hline Students & 0.52 & 0.60 & 0.69 & 0.54 & 0.57 & 0.31 \\
\hline Withdrawals & 1 & 1 & $\ldots$ & 1 & $\ldots$ & $\ldots$ \\
\hline Final test before 12 Dec 2020 & 250 & 109 & 28 & 46 & 35 & 141 \\
\hline
\end{tabular}

Data are presented as No. (\%) unless otherwise indicated.

Abbreviation: SD, standard deviation.

(all different participants) were inconclusive (Table 2). Based on submitted photographs, most LFT results were correctly reported as negative, 5 positive tests were confirmed as positive, but 3 positive tests could not be accurately verified due to unclear photographs. No positive LFT result was incorrectly reported as negative.

Eight positive RT-PCR results (all in different participants) were reported, and 7 of these participants had reported a negative LFT result within the previous week (Supplementary Figure 1). Five who reported a positive LFT result uploaded an RT-PCR test result within 1 week (before or after): 3 of these were positive and 2 were negative (Supplementary Figure 2). All except 1 of those with a positive PCR result, and all except 2 of those with a positive LFT result, stated that they intended to self-isolate.

\section{Symptoms}

App-based symptom tracking was recorded on 2824 occasions by 300 participants (Table 3 ). Of these, symptoms were reported on 29 occasions (1\%) in 17 participants. Cough (9 occasions in 7 participants) and tiredness (8 occasions in 7 participants) were the most frequently reported symptoms. All reported temperatures were $<38^{\circ} \mathrm{C}$.

\section{Acceptability Reported Through App}

All 3187 cases reported acceptability via the app. The majority were acceptable or very acceptable $(n=2907$ [91\%]), 232 (7.3\%) were neutral, and 48 (1.5\%) were unacceptable or very unacceptable (Table 3).

\section{Survey Results}

Participants (213) completed the survey (response rate 29\%) between 1 December 2020 and 7 January 2021. They completed between 1 and 13 tests (mean, 5.8). Sixty-one respondents were undergraduates (28\%), 81 were postgraduate (38\%), and 72 were staff (34\%). Two hundred nine (98\%) reported no COVID-19 symptoms during participation. Overall, respondents reported that self-testing was feasible and acceptable. 


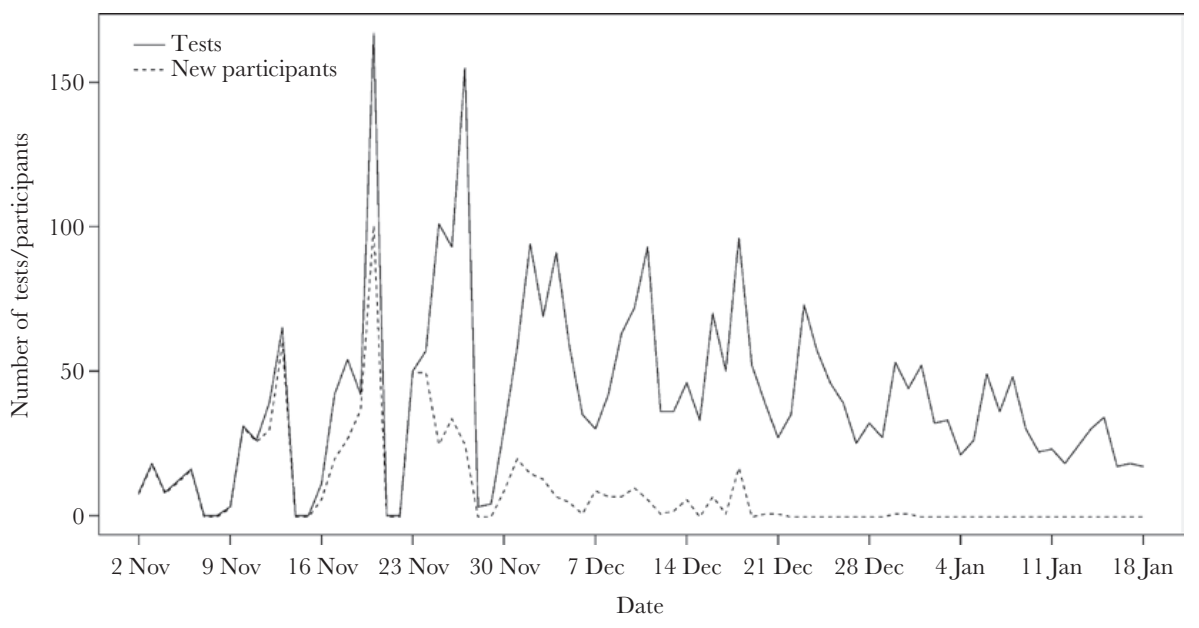

Figure 2. Number of daily lateral flow test results reported (black line) and recruitment rate of participants (dashed line) throughout the study period (80 days).

Self-testing was reported as beneficial for them (97\%), their friends and family $(99.5 \%)$, people they live with $(98 \%)$, and their wider community (98.5\%). Ninety-eight percent felt confident in self-testing abilities and $100 \%$ reported confidence in result interpretation. Fitting self-testing into usual activities (95.7\%) and remembering to test (96\%) were also reported as easy. There was confidence in test safety (99.5\%) and $90 \%$ believed that tests provided reliable and accurate results. Ninetyeight percent reported an intention to self isolate after a positive LFT test, whereas $100 \%$ said they would self-isolate after a positive PCR. Supplementary Table 1 provides a summary of all survey results.

\section{Qualitative-Key Findings (Based on Interviews and Free-Text Comments From the Survey)}

Of those contacted for interview, 431 (59\%) consented, 52 were approached, and 18 were interviewed (response rate $35 \%$ ). Three were undergraduate, 3 were postgraduate, and 12 were staff. Interviews took place between 11 December 2020 and 18 January 2021. Each interviewee completed between 3 and 10 tests during the study period (mean, 7.7).

\section{Table 2. Test Results}

\begin{tabular}{lrrrrr}
\hline & & Site & Site & Site & \\
Result & Overall & A & B & C & Other \\
\hline Total No. of LFTs & & & & & \\
Negative & 3170 & 1041 & 682 & 988 & 459 \\
Positive & 9 & 3 & 4 & 2 & $\ldots$ \\
Inconclusive & 8 & 3 & 4 & 1 & $\ldots$ \\
$\begin{array}{l}\text { Incorrectly interpreted neg- } \\
\quad \text { ative result as positive }\end{array}$ & 3 & $\ldots$ & 3 & $\ldots$ & $\ldots$ \\
Total number of RT-PCR tests & & & & & \\
$\quad$ Positive & 8 & 4 & 2 & 2 & $\ldots$ \\
$\quad$ Negative & 40 & 16 & 2 & 12 & 10 \\
\hline
\end{tabular}

Data are presented as No.

Abbreviations: LFT, lateral flow test; RT-PCR, reverse-transcription polymerase chain reaction.
Regular testing was valued for providing reassurance that participants were not infected, and reduced fear of accidentally infecting family, friends, or others. Additionally, knowing if they were infected so they could take appropriate action was important. While several found testing uncomfortable or even "extremely unpleasant," this did not seem to impact on continued self-testing as participants focused on perceived benefits.

While most viewed the test as providing reassurance about infection status, participants were unsure how accurate the tests are and would have liked more information on the test accuracy, especially in relation to "false negatives."

\section{Table 3. Summary of Symptom and Acceptability Data Collected Through the App}

\begin{tabular}{|c|c|c|c|c|c|}
\hline Symptom & Overall & Site A & Site B & Site C & Other \\
\hline \multicolumn{6}{|l|}{ Daily symptom tracking } \\
\hline Total No. completed & 2824 & 1413 & 1120 & 85 & 206 \\
\hline No. with no symptoms & 2795 & 1393 & 1111 & 85 & 206 \\
\hline $\begin{array}{l}\text { No. that completed symp- } \\
\text { toms at least once }\end{array}$ & 300 & 115 & 88 & 42 & 55 \\
\hline \multicolumn{6}{|c|}{ Symptoms reported, No. of times reported (No. of participants) } \\
\hline Cough & $9(7)$ & $7(5)$ & $2(2)$ & $\ldots$ & \\
\hline Loss of taste or smell & $1(1)$ & & $1(1)$ & $\ldots$ & $\ldots$ \\
\hline Fever & $2\left(1^{\mathrm{a}}\right)$ & $2(1)$ & $\ldots$ & $\ldots$ & \\
\hline Shortness of breath & $4(4)$ & $1(1)$ & $3(3)$ & $\ldots$ & $\ldots$ \\
\hline Tiredness & $8(7)$ & $5(4)$ & $3(3)$ & $\ldots$ & \\
\hline $\begin{array}{l}\text { Unable to do usual ac- } \\
\text { tivities }\end{array}$ & 7 (5) & $1(1)$ & $6(4)$ & $\ldots$ & $\ldots$ \\
\hline \multicolumn{6}{|c|}{ Acceptability of all LFTs conducted } \\
\hline Very acceptable & 1716 & 482 & 431 & 547 & 256 \\
\hline Acceptable & 1191 & 477 & 214 & 331 & 169 \\
\hline Neutral & 232 & 78 & 34 & 91 & 29 \\
\hline Unacceptable & 21 & 6 & 4 & 9 & 2 \\
\hline Very unacceptable & 27 & 4 & 7 & 13 & 3 \\
\hline
\end{tabular}

Abbreviation: LFT, lateral flow test.

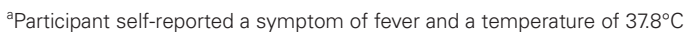


Participants valued the training and information provided, but interviews and survey comments revealed that some felt unsure whether they were swabbing their throat correctly. This in turn made them worry about accuracy of results. Consequently, participants wanted more information on ensuring that they test correctly. Participants who were able to self-test at home, in comparison to those who had to attend a central testing site, reported it easier to take part in regular testing. Full findings from qualitative interviews have been reported separately [16].

\section{DIscussion}

\section{Principal Findings}

FACTS provides the first research evidence on recruitment and training, adherence to, and acceptability of regular COVID19 self-testing for asymptomatic individuals using LFTs. Quantitative and qualitative data show that self-testing was acceptable and results could be correctly interpreted. The survey showed perception of broad benefits to testing for participants and others, and that test accuracy was trusted, but qualitative data revealed that beliefs about test accuracy varied. Interviews highlighted that participants wanted to know their infection status to avoid unintentionally infecting others.

The $25 \%$ participation across the 3 primary sites is well below the government's scientific advisory group's minimum [9]. However, not all invited could take part, for example, those not in residence during study recruitment due to COVID-19 restrictions. Eighty-one percent of participants from the 3 primary sites completed $\geq 2$ tests and $62 \%$ completed $\geq 4$ test, demonstrating that of those who did participate, the majority were willing to undergo further self-testing. Staff had a higher follow-up rate, suggesting that staff were more likely to be compliant to testing and remained in the study for a higher proportion of their potential follow-up time than students. Participants adhered to approximately weekly testing ( 0.96 tests/week overall and 0.91 tests/week in the 3 main sites).

\section{Strengths and Weaknesses of the Study}

This is the first study to assess feasibility and acceptability of regular SARS-CoV-2 self-testing including test result interpretation. FACTS benefited from a mixed-methods evaluation of perceived acceptability and feasibility of regular rapid diagnostic self-testing, highlighting key barriers and facilitators. Triangulation of survey and qualitative data [17] allowed identification of further insights, especially in relation to perceived accuracy and importance of training.

Participation across the 3 main sites ranged from $18 \%$ to $50 \%$ of eligible students and staff, but each site had very different recruitment strategies and different proportions of undergraduate and postgraduate students. Staff, largely working from home, were recruited much later.
During follow-up, there were only 9 positive LFTs and of these, only 5 participants uploaded RT-PCR test results. It was not possible to determine whether the 4 others with a positive LFT had an RT-PCR test but did not upload the result. Symptom reporting was poor, but reminders, which may have improved reporting, were not included in this intervention [18-20] and it was not explored whether there were any associations between test results and recent symptom data.

There was potential for selection bias among those who participated in the survey. This could have led to those who believed that self-testing was valuable and feasible being more likely to participate and complete the survey.

\section{Comparison With Existing Literature}

The survey and interviews highlighted that participants saw testing as equally beneficial for themselves, their family and friends, and the wider community. Participants wanted to know their infection status so they could self-isolate if needed and, overall, found self-testing reassuring. This appeared to be linked to viewing test results as reliable and accurate. However, qualitative data highlighted limited understanding of LFT accuracy, especially around false negatives. While previous studies found similar reasons for wanting to have access to SARSCoV-2 testing, these involved PCR [21] or one-off LFTs [22] rather than repeat self-testing. One study in a university setting previously highlighted the importance of convenience [21]. Our survey and interview data indicate that adherence to repeat selftesting was higher when participants were provided with packs to take home.

Other studies explored the feasibility of university campus testing. One involving students and staff who completed selfswabbing for 2 weeks concluded that this was feasible and acceptable, although laboratory analysis, rather than selfadministered LFTs, was used to obtain results [23]. Another UK study explored the feasibility and acceptability of self-testing with disposable fingerprick blood sample devices [24]. Our work differs by providing data on acceptability of self-swabbing, completing the test, and interpreting results.

\section{Meaning of the Study}

This study indicates that self-testing of asymptomatic staff and students in a university setting is both feasible to implement with low intervention training and acceptable to participants. It has provided real-world evidence on likely uptake, follow-up, and adherence to inform rapid self-testing in universities and similar settings, such as schools or workplaces. We found that $81 \%$ of participants completed at least 2 , and $62 \%$ completed 4 or more, tests over the 5.6-week study period. This is far less than the retesting every 2-3 days suggested to be necessary to keep virus reproduction rates sufficiently low [8]. This work indicated that different techniques, such as the inclusion of reminders or incentives, may need to be investigated to encourage 
some to continue with testing. People's motivations for doing self-testing are complex, and more work is needed to understand how to encourage more optimal frequency of testing.

It has been reported that of those testing positive in UK universities, the majority are compliant with self-isolation guidance. However, in those without a confirmed test, even with symptoms, self-isolation was lower [25]. Therefore, identifying people willing to self-test when asymptomatic, and who proceed to self-isolate, may be challenging and needs to be considered when rolling out testing schemes $[26,27]$. When FACTS began, national COVID-19 prevalence was relatively low. This may have reduced the sense of urgency or concern about contracting or passing on the virus. The study team received informal feedback that some students did not participate because, if they tested positive, their entire household group would have to isolate. Qualitative interviews corroborated this [16]. This is an unintended effect of the national self-isolation guidance, and may influence willingness to participate in future mass testing schemes, particularly among groups who fear a loss of income resulting from self-isolation [28, 29]. Furthermore, incentives may have encouraged adherence; for example, recruitment or follow-up may have increased if those who tested negative were given access to university libraries or face-to-face teaching.

The Innova Antigen Test has been criticized for missing too many infections when not administered by trained health care professionals [30]. Our results suggest that people require accurate information about test performance, especially in relation to false-negative results, as this is a major driver of uptake, adherence, and behavior following a self-test result. It is a possibility that some of the negative LFTs in our study were false negatives. Most of those who had a positive RT-PCR result had performed a negative LFT a few days previously and may later have gone on to develop symptoms. We did not look at accuracy of LFTs, though this has been carried out in another asymptomatic UK university population [31], where LFTs were found to detect $100 \%$ of negative cases but detected lower proportions of positive cases. Authors speculated that many previously reported poor LFT results were due to poor sampling technique rather than diagnostic accuracy. Furthermore, they also suggested that most false negatives were just outside the limit of detection for the Innova LFTs, which may be representative of those at the very early or very late stages of infection. They concluded that it is important to perform regular, routine testing to identify infection before transmission.

\section{CONCLUSIONS}

FACTS indicates that regular self-testing of asymptomatic students and staff in a university setting is both feasible to implement with low intervention training and acceptable to participants. Repeat testing has the potential to interrupt transmission in student populations and could be used along with other measures to control the spread of SARS-CoV-2 when students return to residential campuses [32]. However, testing alone will not prevent transmission and quarantining for those who test positive would need to be followed for it to control transmission [33]. It is therefore important to rigorously assess the strategy, as well as the test, with high-quality studies to ensure it is effective and cost-effective for preventing infections.

\section{Supplementary Data}

Supplementary materials are available at Open Forum Infectious Diseases online. Consisting of data provided by the authors to benefit the reader, the posted materials are not copyedited and are the sole responsibility of the authors, so questions or comments should be addressed to the corresponding author.

\section{Notes}

Author contributions. F. D. R. H., J. J. L., M. L., B. D. N., R. P., S. T.-C., and M. W. devised the study. F. D. R. H. acquired the funding. F. D. R. H., M. L., J. A. H., B. D. N., I. T., G. D., P. T., C. V., and M. B. managed the planning and running of the study. T. R. F., L. M., and R. P. analyzed the data. J. A. H., M. L., T. R. F., L. M., S. T.-C., and M. W. co-wrote the first draft of the manuscript, and all authors provided critical feedback and helped shape the research, analysis, and final manuscript.

Acknowledgments. The Feasibility and Acceptability of Community Coronavirus Disease 2019 Testing Strategies (FACTS) study management group and training team: Kirsty Jackson, Moscho Michalopoulou, Stuart Faulkner, Johanna Cook, Barbara Robinson, Sarah Brown, Georgie Harmer, Thomas Gabriel, Anisa Hajizadeh, Claire Friedemann Smith, Nerys Asbury, Ailsa Butler, George Edwards, Nick Fahy, Elif Coker, Mina Davoudianfar, and Esther van Vliet. The authors acknowledge Merton College and staff; St Hilda's College and staff; Cammy Crolic, Peter Drobac, and Andrew Stephen from the Said Business School; and Chris Maynard (Senior Product Manager) and Jardine Ian Barrington-Cook (Tech Lead) at Sensyne Health PLC.

Patient consent statement. Each participant in this study submitted their consent via the bespoke study app prior to the start of their participation. This study was approved by the University of Oxford Research Ethics Committee (CUREC ethics reference R72896/RE001 October 2020).

Disclaimer. The views expressed are those of the author(s) and not necessarily those of the National Health Service (NHS), the National Institute for Health Research (NIHR), or the UK Department of Health and Social Care.

Financial support. This work was funded by the Oxford University Medical Sciences Division. Data collection and analysis were done by university staff, but without input from the funders. M. L. and J. A. H. are funded by the NIHR Biomedical Research Centre, Oxford. T. F. and L. M. receive funding from the NIHR Community Healthcare MedTech and In Vitro Diagnostics Co-operative at Oxford Health NHS Foundation Trust. T. F. receives funding from the NIHR Applied Research Collaboration Oxford and Thames Valley at Oxford Health NHS Foundation Trust. S. T.-C. is funded by the NIHR Health Protection Research Unit in Healthcare Associated Infections and Antimicrobial Resistance, University of Oxford, in partnership with Public Health England. L. T. also acknowledges partial support from the NIHR Biomedical Research Centre, Oxford. B. D. N. is funded by an NIHR Academic Clinical Lectureship. F. D. R. H. acknowledges partial support from the NIHR School for Primary Care Research, the NIHR Collaboration for Leadership in Applied Research in Health and Care Oxford, and the NIHR Biomedical Research Centre, Oxford.

Potential conflicts of interest. L. T. works part-time for Sensyne Health as Research and Development Director and holds share options in the company. He also reports a research grant and personal fees from the company. All other authors report no potential conflicts of interest.

All authors have submitted the ICMJE Form for Disclosure of Potential Conflicts of Interest. Conflicts that the editors consider relevant to the content of the manuscript have been disclosed. 


\section{References}

1. He X, Lau EHY, Wu P, et al. Temporal dynamics in viral shedding and transmissibility of COVID-19. Nat Med 2020; 26:672-5.

2. Peto J, Alwan NA, Godfrey KM, et al; 27 signatories. Universal weekly testing as the UK COVID-19 lockdown exit strategy. Lancet 2020; 395:1420-1.

3. UK Parliament. Mass testing for COVID-19 using lateral flow tests 2020. https:// post.parliament.uk/mass-testing-for-covid-19-using-lateral-flow-tests/. Accessed 5 February 2021.

4. Andryukov BG. Six decades of lateral flow immunoassay: from determining metabolic markers to diagnosing COVID-19. AIMS Microbiol 2020; 6:280-304.

5. Iacobucci G, Coombes R. Covid-19: government plans to spend $£ 100$ bn on expanding testing to 10 million a day. BMJ 2020; 370:m3520.

6. Rudolf F, Kaltenbach H-M, Linnik J, et al. Clinical characterisation of eleven lateral flow assays for detection of COVID-19 antibodies in a population. medRxiv [Preprint]. Posted online 25 August 2020. doi: 10.1101/2020.08.18.20177204.

7. World Health Organization. Target product profiles for priority diagnostics to support response to the COVID-19 pandemic version 1.0. 2020. https://www. who.int/docs/default-source/blue-print/who-rd-blueprint-diagnostics-tpp-finalv1-0-28-09-jc-ppc-final-cmp92616a80172344e4be0edf315b582021.pdf?sfvrsn=e 3747f20_1\&download=true. Accessed 9 February 2021.

8. Paltiel AD, Zheng A, Walensky RP. Assessment of SARS-CoV-2 screening strategies to permit the safe reopening of college campuses in the United States. JAMA Netw Open 2020; 3:e2016818.

9. UK Government. TFMS: behavioural paper supporting the consensus statement on mass testing. 2020. https:/www.gov.uk/government/publications/ tfms-behavioural-paper-supporting-the-consensus-statement-on-mass-testing27-august-2020. Accessed 8 March 2021.

10. UK Government. Multidisciplinary task and finish group on mass testing. Consensus statement for SAGE 2020. https://assets.publishing.service.gov.uk/ government/uploads/system/uploads/attachment_data/file/914931/s0712-tfmsconsensus-statement-sage.pdf. Accessed 8 March 2021.

11. Davies NG, Klepac P, Liu Y, et al; CMMID COVID-19 Working Group. Agedependent effects in the transmission and control of COVID-19 epidemics. Nat Med 2020; 26:1205-11.

12. Yamey G, Walensky RP. Covid-19: re-opening universities is high risk. BMJ $\mathbf{2 0 2 0}$ 370:m3365.

13. Innova Medical Group. About Innova SARS-CoV-2 Rapid Antigen Test. https:// innovamedgroup.com/innova-rapid-antigen-test/.

14. Fusch PI, Ness LR. Are we there yet? Data saturation in qualitative research. Qualitative Rep 2015; 20:1408-16.

15. Johnson GA, Vindrola-Padros C. Rapid qualitative research methods during complex health emergencies: a systematic review of the literature. Soc Sci Med 2017; 189:63-75.

16. Wanat M, Logan M, Hirst JA, et al. Perceptions on undertaking regular asymptomatic self-testing for COVID-19 using lateral flow tests: a qualitative study of university students and staff. BMJ Open 2021; 11:e053850.

17. Denzin NK. Triangulation 2.0. J Mixed Methods Res 2012;6:80-8.
18. Haider R, Sudini L, Chow CK, Cheung NW. Mobile phone text messaging in improving glycaemic control for patients with type 2 diabetes mellitus: a systematic review and meta-analysis. Diabetes Res Clin Pract 2019; 150:27-37.

19. Normansell R, Kew KM, Stovold E. Interventions to improve adherence to inhaled steroids for asthma. Cochrane Database Syst Rev 2017; 4:CD012226.

20. Robotham D, Satkunanathan S, Reynolds J, et al. Using digital notifications to improve attendance in clinic: systematic review and meta-analysis. BMJ Open 2016; 6:e012116.

21. Blake H, Corner J, Cirelli C, et al. Perceptions and experiences of the university of Nottingham pilot SARS-CoV-2 asymptomatic testing service: a mixed-methods study. Int J Environ Res Public Health 2020; 18:188. doi:10.3390/ijerph18010188.

22. University of Liverpool. Liverpool COVID-19 Community Testing Pilot. Liverpool, UK: University of Liverpool; 2020.

23. Gillam TB, Cole J, Gharbi K, et al. Norwich COVID-19 testing initiative pilot: evaluating the feasibility of asymptomatic testing on a university campus. J Public Health (Oxf) 2021; 43:82-8.

24. Atchison C, Pristerà $\mathrm{P}$, Cooper E, et al. Usability and acceptability of home-based self-testing for severe acute respiratory syndrome coronavirus 2 (SARS-CoV-2) antibodies for population surveillance. Clin Infect Dis 2021; 72:e384-93.

25. Nixon E, Trickey A, Christensen H, et al. Contacts and behaviours of university students during the COVID-19 pandemic at the start of the 2020/2021 academic year. Sci Rep 2021; 11:11728.

26. Gill M, Gray M. Mass testing for Covid-19 in the UK. BMJ 2020; 371:m4436

27. Mahase E. Covid-19: people are not being warned about pitfalls of mass testing. BMJ 2021; 372:n238.

28. Hamza CA, Ewing L, Heath NL, Goldstein AL. When social isolation is nothing new: A longitudinal study psychological distress during COVID-19 among university students with and without preexisting mental health concerns. Canadian Psychology/Psychologie canadienne 2020; 62:20-30.

29. Waugh P. NHS test and trace chief admits workers fear "financial" hit if they self-isolate. 2020. https://www.huffingtonpost.co.uk/entry/dido-hardingself-isolation-financial-worry_uk_5f036a9cc5b612083c5ffc8d?ncid=other huffpostre_pqylmel2bk8\&utm_campaign=related_articles\&guce_referrer $=\mathrm{a}$ HR0cHM6Ly9ibG9ncy5sc2UuYWMudWsv\&guce_referrer_sig=AQAAAGj UJEj7iTmbyTL7F55sleFEZJUGcD4Zs9osMFstHe_Hu7M-\&_guc_consent_ skip=1605190198. Accessed 18 March 2021.

30. Deeks JJ, Raffle AE. Lateral flow tests cannot rule out SARS-CoV-2 infection. BMJ 2020; 371:m4787.

31. Ferguson J, Dunn S, Best A, et al. Validation testing to determine the sensitivity of lateral flow testing for asymptomatic SARS-CoV-2 detection in low prevalence settings: testing frequency and public health messaging is key. PLoS Biol 2021; 19:e3001216.

32. Chang JT, Crawford FW, Kaplan EH. Repeat SARS-CoV-2 testing models for residential college populations. Health Care Manag Sci 2021; 24:305-18.

33. Peto J, Carpenter J, Smith GD, et al. Weekly COVID-19 testing with household quarantine and contact tracing is feasible and would probably end the epidemic. R Soc Open Sci 2020; 7:200915. 